\title{
Lipid profile studies in long term thiazide treated hypertensives
}

\author{
R. Ashok Chandra Rao, B.M. Hegde, E. Keshav Bhat, U. Vidyavathi and \\ R. Raghunandana Rao
}

Department of Medicine, Kasturba Medical College, Mangalore, Karnataka 575 001, India

\begin{abstract}
Summary: Serum cholesterol and its subfractions were estimated in 23 patients on thiazide diuretics, for treatment of mild hypertension, for at least 30 months. The results were compared with those obtained in a matched set of 25 untreated mild hypertensives. It was found that while there was no difference in the level of total cholesterol, the LDL-cholesterol was significantly lower and HDL-cholesterol significantly higher in the treated group. It was also found that the total:LDL-cholesterol ratio was more in the treated group and the total:HDL-cholesterol ratio was less in the treated group. These findings suggest that coronary risk attributable to cholesterol and its subfractions does not seem to operate in long term thiazide treated patients.
\end{abstract}

\section{Introduction}

Since the publication of the results of the Multiple Risk Factor Intervention Trial (MRFIT),${ }^{1} \mathrm{a}$ lot of interest has been generated in the metabolic effects of thiazide therapy. Many earlier studies, ${ }^{2-6}$ have shown changes in lipid profile thought to be detrimental to the patient, after short term therapy. Some, however, believe that after long term diuretic therapy, the cholesterol levels which had gone up initially, fall to or below pre-treatment values. ${ }^{7,8}$ In the Systolic Hypertension in the Elderly Program, ${ }^{9}$ diuretics did not increase cholesterol levels. Samuel et al.$^{10}$ have shown that there is an improvement in lipid profile after 18 months treatment with pindolol and hydrochlorothiazide (HCTZ) in mild hypertensives.

We have studied the effects of long term thiazide diuretic therapy (for more than 30 months) on serum cholesterol and its subfractions in mild hypertensives and have obtained some interesting data.

\section{Materials and methods}

All subjects were selected for this study from the Wenlock District Hospital, Mangalore, during the

This study was supported in part by a grant from the Karnataka, State Council for Science and Technology, Indian Institute of Science, Bangalore.

Correspondence: Professor B.M. Hegde, M.D., F.R.C.P. Accepted: 18 February 1991 period September 1985 to November 1986. They belonged to the age group 45-70 years. Patients who were known diabetics, or had a history/family history of hyperlipidaemic states or had a history of any illness which is associated with changes in lipid profile were not considered for the study. Patients found to have diabetes mellitus were excluded. Since all females in the study ( 3 in the treated group and 2 in the untreated) were in the post-menopausal state, they were not considered as a special group. Patients diagnosed or suspected to have secondary hypertension were not selected.

The thiazide treated group consisted of a set of 23 patients receiving thiazide diuretics for at least 30 (60 \pm 18$)$ months (ascertained by thorough history and from treatment records). Three of these had, at some time in the remote past, received some other antihypertensive drug, but all had a thiazide as the fundamental antihypertensive agent. Hydrochlorothiazide (HCTZ) was the commonest diuretic (15 patients; dose $34 \pm 16.2 \mathrm{mg} /$ day) used. Chlorthalidone $(100 \mathrm{mg} /$ day) was used in 6 , polythiazide $(1 \mathrm{mg} /$ day $)$ in one and benzthiazide $(2.5$ $\mathrm{mg} /$ day) in another. No patient received potassium supplementation.

The untreated group consisted of a set of 25 freshly detected hypertensives. They had a systolic blood pressure less than $200 \mathrm{mmHg}$ and a diastolic blood pressure in the range of $90-114 \mathrm{mmHg}$ in both supine and upright position. Blood pressure was checked on 3 separate occasions using a mercury sphygmomanometer (diastolic phase V).

Patients in the 2 groups were matched for age, sex, body mass index, dietary habits, alcohol intake 
and smoking habits.

Using a blood sample obtained (with precautions to prevent haemolysis) after 12-14 hours of overnight fasting, the following estimations were done: serum total cholesterol, high density lipoprotein (HDL)-cholesterol, low density lipoprotein (LDL)-cholesterol, and serum uric acid and potassium.

Zak's ${ }^{11}$ method was used for estimation of serum cholesterol. LDL-cholesterol and HDL-cholesterol were estimated after precipitation of LDL-cholesterol using phosphotungstate-magnesium chloride mixture $;{ }^{11}$ from these values, the various ratios were calculated. Serum uric acid and potassium were measured by routine methods.

HDL-2 was estimated in 12 randomly selected treated and 10 well matched untreated patients. For this purpose, $2 \mathrm{ml}$ plasma was stored in the frozen state. After all the samples were collected, they were air lifted (in the frozen state) to Bombay, and the estimation done at the CVTC Laboratory, K.E.M. Hospital there.

The mean values in the treated and untreated groups were analysed using the 'unpaired $t$-test'.

\section{Results}

The values obtained in the 2 groups are shown in Table I. On comparing the data in the treated and control groups, it was found that the mean values of HDL-cholesterol were $29 \%$ higher and LDLcholesterol $10.4 \%$ lower in the treated group. The total cholesterol:LDL-cholesterol ratio was $10.9 \%$ higher while the total cholesterol:HDL-cholesterol and LDL-cholesterol:HDL-cholesterol ratios were $19.1 \%$ and $26.2 \%$ lower, respectively, in the treated group. All the above mentioned changes are statistically significant (Table I). The mean value of total cholesterol was not significantly altered in the two groups while that of HDL 2 (though in a smaller number of patients) was significantly higher in the thiazide treated group.

\section{Discussion}

Samuel et al..$^{10}$ have brought out data to show that there is an improvement in the lipid profile after long term administration of pindolol and HCTZ to mild hypertensives. Earlier studies ${ }^{2-6}$ had shown that after short term therapy with thiazides alone, changes occurred in lipid profile considered harmful in the evaluation of risk factors for coronary artery disease. Samuel et al. concluded that the improvement in lipid profile was due to the fact that thiazide diuretic was combined with pindolol. However, Marvin Moser, reviewing the clinical trials in the field of mild hypertension, observed that after long term therapy, the serum cholesterol levels were not higher than pretreatment levels in patients receiving thiazide diuretics. ${ }^{8}$

In our study, it was found that hypertensive patients on long term thiazide therapy (more than 30 months) had a more favourable lipid profile than matched untreated hypertensives, in the assessment of lipid risk factors for coronary artery disease. Our results show that the total:LDLcholesterol, total:HDL-cholesterol ratios were not unfavourable in the thiazide treated patients nor

Table I Summary of the results and statistical data

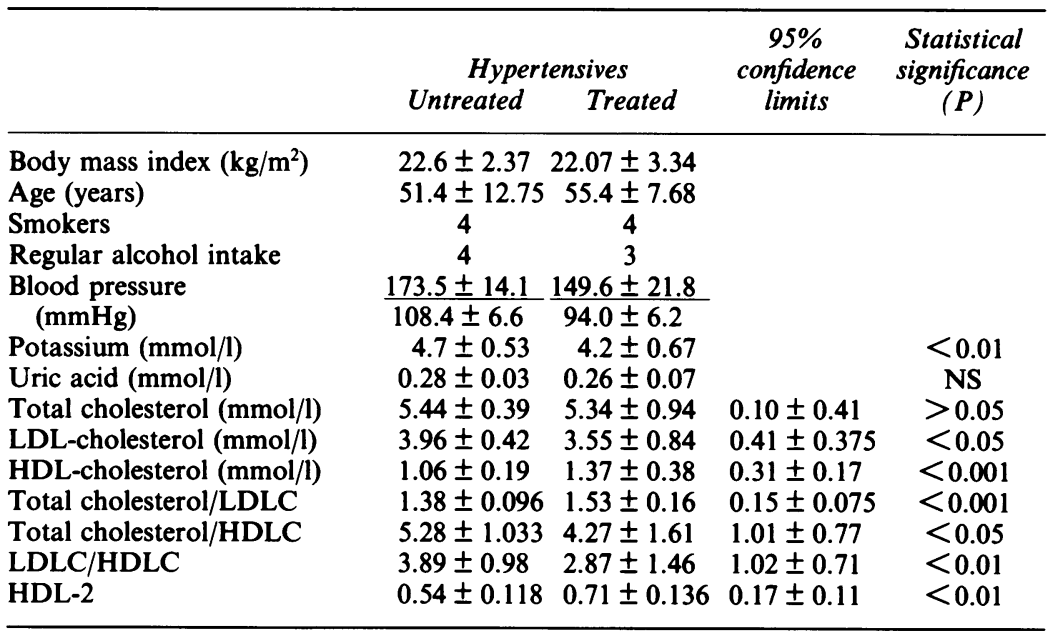

NS - not significant. 
were the absolute values of cholesterol or any of its subfractions in the assessment of risk factors for coronary artery disease. ${ }^{14}$ Further, HDL-2 values were found to be higher in the treated group by $31 \%(P<0.01)$.

It has been suggested by Samuel $e t$ al. that the changes observed by them are probably due to the effects of pindolol on the enzyme lecithin-cholesterol acyl transferase. But any effect observed after a very long term treatment is most unlikely to be due to the direct effect on enzyme function. Further, in our study, patients did not receive pindolol. Moser, in his recent review of trials involving the treatment of mild hypertension by thiazide diuretics, did not find any deterioration in lipid profile after long term (more than one year) treatment. ${ }^{8}$ It is difficult to explain the mechanisms underlying the changes in lipid profile under these circumstances and the results of our study. However, the crucial step in LDL metabolism is receptor mediated uptake. ${ }^{13}$ It is tempting to speculate that the

\section{References}

1. Multiple Risk Factor Intervention Trial Group: Multiple Risk Factor Intervention Trial: Risk factor changes and mortality results. $J A M A$ 1982, 248: $1465-1477$.

2. Ames, R.P. \& Hill, P. Elevation of serum lipids during diuretic therapy for hypertension. Am J Med 1976, 61: 748-757.

3. Ames, R.P. \& Hill, P. Increase in serum lipids during treatments of hypertension with chlorthalidone. Lancet 1976, i: $721-723$.

4. Goldmann, A.I., Steele, B.W., Schnaper, H.W., Eitz, A.E., Frolich, E.D. \& Perry, H.M. Jr. Serum lipoprotein levels during chlorthalidone therapy. JAMA 1980, 244: 1691-1695.

5. Aldermann, M.V., Davies, T.K. \& Carrol, R.N. Initial antihypertensive therapy. Am J Med 1986, 80 (Suppl 2A): 120-125.

6. Grimm, R.H. Jr., Leon, A.S., Hunninghake, D.B., Lenz, K., Hannan, P. \& Blackburn, H. Effects of thiazide diuretics on plasma lipids and lipoproteins in mildly hypertensive patients: a double blind controlled trial. Ann Intern Med 1981, 94: 7-11.

7. Moser, M., Diuretics in the management of hypertension. Med Clin North Am 1987, 71: 935-946. favourable changes in lipid profile seen in our patients on long term thiazides, could be due to alterations in receptor function induced by the drug. We do not believe that dietary changes were responsible.

It is very clear that the lipid profile of thiazidetreated hypertensives is in no way worse and may in fact, be better than that of matched untreated hypertensives. Thiazide diuretics are a boon to the multitude of poor patients in the third world as they are effective in more than $80 \%$ of mildmoderate hypertensive patients, ${ }^{14}$ and are relatively inexpensive.

\section{Acknowledgement}

The authors wish to thank Dr (Mrs) T.S. Tasker, Reader in Biochemistry, CVTC Laboratory, KEM Hospital, Bombay for HDL-2 estimations.

8. Moser, M. Lipid abnormalities and diuretics. A F P 1989, 40: 213-220.

9. Hully, S.B., Furberg, C.D., Gurland, B. et al. For the SHEP? Research Group. Systolic Hypertension in the Elderly Pro-gram (SHEP): Anti-hypertensive efficacy of chlorthalidone. Am J Cardiol 1985, 56: 913-920.

10. Samuel, P., Chin, B., Fenderson, R.W., Schoenfeld, B.H., Gonasun, L.M. \& Lieberman, S. Improvement of the lipid profile during long-term administration of pindolol and hydrocholorothiazide in patients with hypertension. Am J Cardiol 1986, 57: 24C-28C.

11. Varley, H., Gowenlock, A.H. \& Bell, M. (eds). Practical Clinical Biochemistry, vol. 1. William Heinemann, London, 1980 , pp. 665-680.

12. Oliver, M.F. What should I do for mild hypercholesterolaemia? Proc $R$ Coll Surg Edinb 1987, 17: 105-106.

13. Brown, S., Kovanen, P.T. \& Goldstein, J.L. Regulation of plasma cholesterol by lipoprotein receptors. Science 1989 , 212: $628-635$.

14. Beevers, D.G., Hamilton, M. \& Harpur, J.E. The long-term treatment of hypertension with thiazide diuretics. Postgrad Med J 1971, 47: 639-643. 